\title{
Low-frequency variability of the Kuroshio Extension
}

\author{
S. Pierini ${ }^{1}$ and H. A. Dijkstra ${ }^{2}$ \\ ${ }^{1}$ Dipartimento di Scienze per l'Ambiente, Università di Napoli Parthenope, Naples, Italy \\ ${ }^{2}$ Institute for Marine and Atmospheric research Utrecht, Department of Physics and Astronomy, Utrecht University, \\ Utrecht, The Netherlands
}

Received: 20 July 2009 - Revised: 27 October 2009 - Accepted: 12 November 2009 - Published: 14 December 2009

\begin{abstract}
In this paper, we provide a review of recent results targeted at the understanding of the low-frequency variability of the Kuroshio Extension. We provide the background and main arguments of two views which have recently been proposed to explain this variability. In the first view, windinduced Rossby waves and the effects of mesocale eddies are crucial. The second view is based on low-dimensional equivalent-barotropic large-scale nonlinear dynamics, with neither Rossby wave dynamics nor baroclinic instability being important. Results from models supporting each view are discussed and confronted with results from available observations.
\end{abstract}

\section{Introduction}

There has been much progress over the last decade to understand the peculiar behavior of the Kuroshio Extension (KE hereafter) near the coast of Japan. Since the early 70s, it was recognized that the KE switches between two states: a zonally elongated, fairly stable, energetic meandering jet and a much weaker, very variable and convoluted jet with a reduced zonal penetration (Taft, 1972). Although different terminology has been used to indicate both states, we will refer to them below as "elongated" and 'contracted' state, respectively.

Many more details on the current path and the (local and remote) forcing of the flow have been obtained through the analysis of satellite data (in particular altimetry) and data from the Kuroshio Extension System Study (Donohue et al., 2008), see also www.uskess.org. In Qiu and Chen (2005), bi-weekly paths of the Kuroshio, represented by the $170-\mathrm{cm}$ sea surface height (SSH) contour, were determined over the years 1993-2004. From 1993-1995 and from 2000-2004, the KE was in the elongated state, and from 1995-2000 in

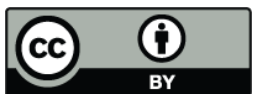

Correspondence to: H. A. Dijkstra (dijkstra@phys.uu.nl) the contracted state. This suggests decadal variations of the KE extension, which is on a much larger time scale than that of mesoscale eddy variability (months).

This low-frequency variability of the KE has also been found in eddy-resolving general circulation models (ERGCMs). In Taguchi et al. (2005), the response of the KE to changes in remote wind stress is studied in a high-resolution $\left(1 / 12^{\circ}\right.$ horizontally) version of the POM model. A hindcast of KE variability over the period $1950-2003$ with the $1 / 10^{\circ}$ global OFES model is analyzed in Taguchi et al. (2007). To understand the observations and results of ER-GCMs, in particular the origin of the bimodal behavior, much effort has been devoted to the development of conceptual models. This has lead to two views of dominant processes controlling the low-frequency variability of the KE.

In the first view, changes in external wind forcing are thought to be crucial, with modifications of the Rossby wave response through mesoscale eddies. The other view is derived from studies with idealized (equivalent) barotropic wind-driven ocean models, in particular on so-called doublegyre flows. Under a steady wind stress these model results show that internal decadal variability can appear spontaneously. This variability is caused by oscillatory instabilities of the mean flow (Jiang et al., 1995; Speich et al., 1995; Dijkstra and Katsman, 1997) and transitions associated with a homoclinic bifurcation (Meacham, 2000; Nadiga and Luce, 2001; Simonnet et al., 2005).

A reasonably successful data-model comparison was presented in Pierini (2006) using an equivalent barotropic shallow-water model under a steady wind forcing. The origin of the low-frequency variability was investigated in Pierini et al. (2009) by studying the flow behavior from the relatively viscous regime to the more inertial regime. It was shown that the low-frequency variability of the KE in the model was caused by homoclinic bifurcations. While the present paper provides no new results, its aim is to provide a short review of recent ideas on the processes controlling KE variability, with focus on explaining in more detail the nonlinear dynamics view. In Sect. 2, we briefly review the Rossby wave view

Published by Copernicus Publications on behalf of the European Geosciences Union and the American Geophysical Union. 


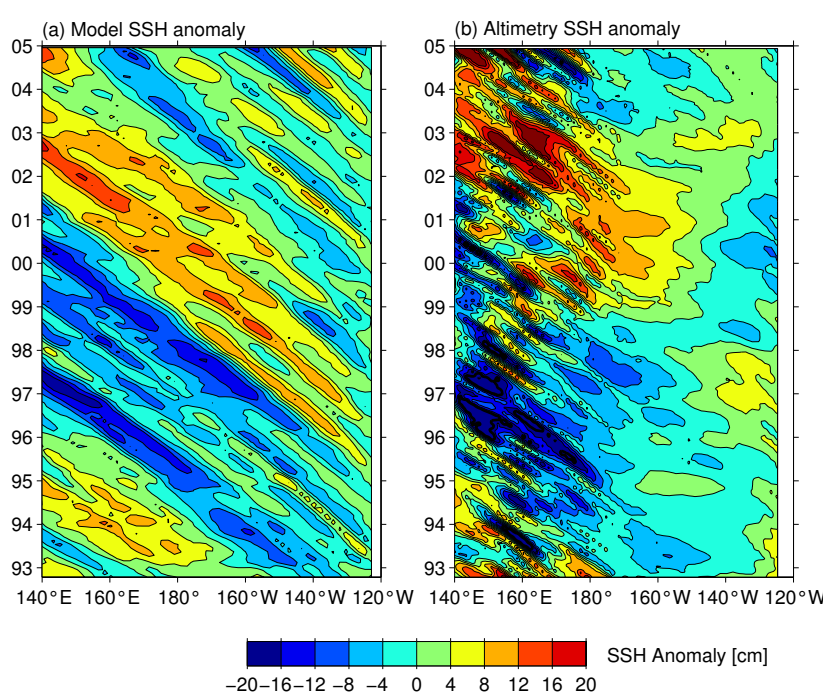

Fig. 1. SSH anomalies along the zonal band of $32^{\circ} \mathrm{N}-34^{\circ} \mathrm{N}$ from (a) the linear vorticity model (1) and (b) the satellite altimetric data (from Qiu and Chen, 2005).

and its main arguments. The successive bifurcation view is presented in Sect. 3 providing details on the occurrence of multiple equilibria, periodic orbits, and the homoclinic transition. Finally, in Sect. 4, we critically compare the two views of low-frequency KE variability and suggest how they possibly can be reconciled.

\section{The Rossby wave view}

In this view, wind stress changes over the North Pacific are thought to be crucial for the transitions between the elongated and contracted state. The wind stress over the North Pacific varies on a decadal time scale, for example, associated with the Pacific Decadal Oscillation (PDO) which has its center of action around $160^{\circ} \mathrm{W}$ (Mantua et al., 1997). When the PDO index is positive (negative), the Aleutian Low shifts southwards (northwards) and negative (positive) SSH anomalies are generated through Ekman divergence. Once generated, they propagate westward at the speed $c_{R}$ of baroclinic Rossby waves which, at typical KE latitudes $\left(\sim 30^{\circ} \mathrm{N}\right)$, is about $4 \mathrm{~cm} / \mathrm{s}$.

The linear vorticity equation under the longwave approximation of the SSH anomaly field $h^{\prime}(x, y, t)$ in local Cartesian coordinates $(x, y)$ is given by

$\frac{\partial h^{\prime}}{\partial t}-c_{R} \frac{\partial h^{\prime}}{\partial x}=-\frac{g^{\prime}}{\rho_{0} g f}\left(\frac{\partial \tau^{x}}{\partial y}-\frac{\partial \tau^{y}}{\partial x}\right)$

where $g$ is the gravitational acceleration, $g^{\prime}$ the reduced gravity, $\rho_{0}$ a reference density, $f$ the Coriolis parameter and $\left(\tau^{x}, \tau^{y}\right)$ the wind-stress field. When the wind stress is given, the SSH anomaly field can be determined along Rossby wave (a) Model SSH
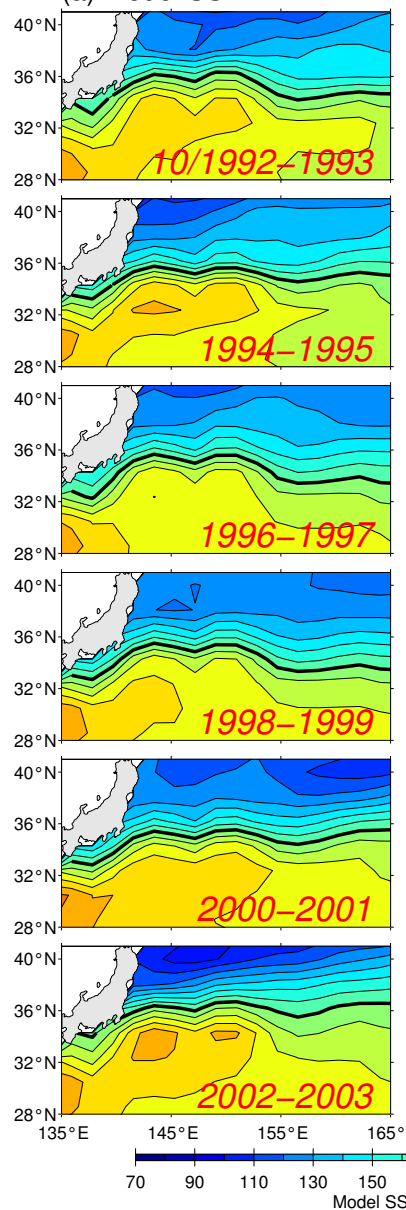

(b) Altimetry $\mathrm{SSH}$
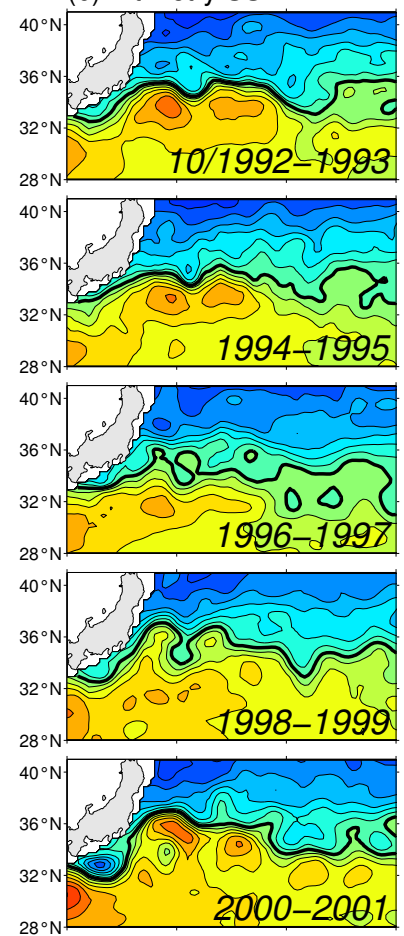

$28^{\circ} \mathrm{N}$

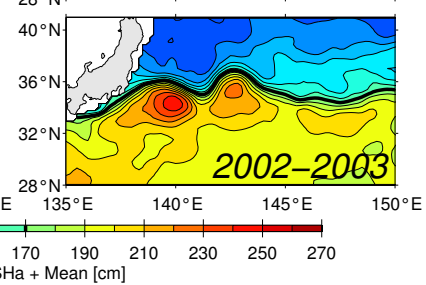

Fig. 2. Biennially averaged SSH fields with the SSH anomalies (a) hindcast by the linear vorticity model and (b) observed by the satellite altimeters. The mean SSH field in (a) is based on Teague et al. (1990) and thick lines denote the 170-cm contours (from Qiu and Chen, 2005).

characteristics. This response was calculated in Qiu and Chen (2005) and is shown here as Fig. 1a, together with the observed SSH anomaly field from altimeter data (Fig. 1b). Indeed, variations in the large-scale wind-stress forcing lead to Rossby waves and large-scale SSH anomalies with similarities between model results and observations in $x-t$ diagrams. The amplitudes of the observed SSH anomalies, however, are larger than those from the Rossby wave model (1) and show variability on smaller scales.

When the SSH anomalies from the Rossby-wave model (1) are superimposed on the mean dynamic topography (Teague et al., 1990), the biennially averaged SSH fields as in Fig. 2a are obtained. Comparison with the corresponding fields derived from altimeter data (Fig. 2b) shows that the intensity of the modeled recirculation gyre south of the main axis of the jet (thick black line) appears in phase with the alternation of the elongated and contracted states of the KE. 
However, the modeled gyre is always much weaker than the observed one and, what is most striking, the jet axis and the nearby isolines are virtually time independent in the model results, while they undergo substantial changes during the observed bimodal cycle. It is clear from these results that additional processes are needed to explain the bimodality of the KE. More recently, Qiu and Chen (2009, in press) showed that the mesocale eddy field may induce the large SSH anomalies. From observations, it is found that the eddykinetic energy is much higher in the contracted state than in the elongated state. The eddies arise through instability of the jet and may induce upgradient transport of momentum giving large SSH anomalies.

To summarize this view, Rossby waves generated by timevarying winds produce a spatially broad variability in the KE region which is in synchrony with the much stronger, spatially sharper bimodal variability observed in altimeter data. Apart from any consideration concerning possible interactions between the two kinds of low-frequency variability (see Sect. 4), it is clear that wind-driven Rossby waves can by no means account for the vigorous bimodal variability so clearly evidenced in observations.

\section{The intrinsically generated variability view}

In this view, the KE bimodal variability is generated through dynamical mechanisms internal to the ocean system. In order to unequivocally recognize the intrinsic origin of the lowfrequency variability in a particular model, a stationary wind field is used as forcing. In this case, all changes found in the equilibrium (asymptotic) response are necessarily due to mechanisms internal to the model ocean.

Starting point is the theory of the homogeneous steady wind-driven ocean circulation (Sverdrup, 1947; Stommel, 1948; Munk, 1950) which is one of the cornerstones in physical oceanography. One of the simplest situations within this theory is that of an active layer of ocean water with constant density $\rho$ located in a rectangular ocean basin. Below this layer, with equilibrium thickness $H$, there is a very deep motionless layer of density $\rho+\Delta \rho$. The flows are considered on a midlatitude $\beta$-plane with Coriolis parameter $f=f_{0}+\beta y$.

\subsection{The equivalent-barotropic quasi-geostrophic model}

Let the flow be characterized by a horizontal length scale $L$ and a horizontal velocity scale $U$. When the Rossby number $\epsilon=U /\left(f_{0} L\right)$ is small, quasi-geostrophic theory is an adequate description of the large-scale flow (Pedlosky, 1987). Let $\psi$ indicate the geostrophic streamfunction in the horizontal plane, then the zonal velocity $u$, the meridional velocity $v$ and the vorticity $\zeta$ are given by $u=-\partial \psi / \partial y, v=\partial \psi / \partial x$ and $\zeta=\partial v / \partial x-\partial u / \partial y=\nabla^{2} \psi$, respectively. When the flow is driven by a zonal wind stress $\tau$, the governing equation in this theory is the (equivalent) barotropic vorticity equation, given by

$$
\begin{aligned}
\frac{\partial q}{\partial t}+J(\psi, q) & =A_{H} \nabla^{4} \psi+\frac{(\nabla \times \boldsymbol{\tau})_{z}}{\rho H} \\
q & =\zeta-\frac{f_{0}^{2}}{H g^{\prime}} \psi+\beta y
\end{aligned}
$$

Here, $q$ is the potential vorticity, $g^{\prime}=g \Delta \rho / \rho$ is the reduced gravity, $H$ the active layer thickness and the Jacobian operator $J$ is defined as $J(F, G)=F_{x} G_{y}-F_{y} G_{x}$ where the subscripts indicate differentiation. The quantity $A_{H}$ represents the turbulent lateral friction coefficient. A linear damping term, representing interfacial friction in this equivalentbarotropic model, is neglected

In the so-called double-gyre case, flows are considered in a rectangular $L \times B$ basin where the wind-stress forcing is chosen as

$\tau^{x}(x, y)=-\tau_{0} \cos 2 \pi \frac{y}{B} ; \tau^{y}(x, y)=0$

with $\tau_{0}$ a typical amplitude. In this case, the wind stress is symmetric with respect to the mid-axis of the basin. No-slip boundary conditions are usually prescribed at the east-west boundaries and slip conditions at the north-south boundaries, i.e.,

$x=0, L: \psi=0, \quad \frac{\partial \psi}{\partial x}=0$

$y=0, B: \psi=0, \quad \zeta=0$

Under a given steady wind-stress forcing, the linear steady quasi-geostrophic theory (neglecting the term $J(\psi, q)$ ) predicts a Sverdrup interior flow and a frictional western boundary layer. The linear theory provides a first order explanation of the existence of western boundary currents, such as the Kuroshio. The nonlinear theory is, however, still far from complete. Although the strong effects of inertia on the flows was already shown by Veronis (1963), the work to determine systematically the solution structure of (2a) versus the lateral friction parameter $A_{H}$ did not start until the mid 1990s (Cessi and Ierley, 1995; Dijkstra and Katsman, 1997).

\subsection{Primary bifurcations}

For large values of $A_{H}$, a unique and globally stable flow state for both single- and double-gyre cases is found (Dijkstra and De Ruijter, 1996). To investigate the solution structure of the equations when $A_{H}$ is decreased, continuation methods (Dijkstra, 2000) have been used on discretized versions of $(2 \mathrm{a}-4 \mathrm{~b})$.

The structure of the steady solutions is shown through the bifurcation diagram in Fig. 3, where the value of the streamfunction at a point in the southwest part of the domain $\left(\psi_{S W}\right)$ is plotted versus $R e=U L / A_{H}$. At large values of $A_{H}$ 


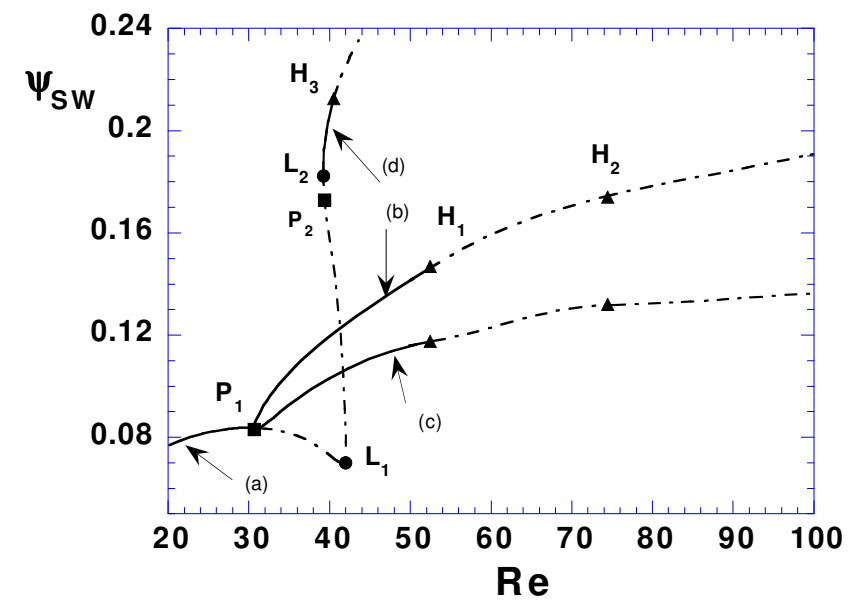

Fig. 3. Bifurcation diagram for the double-gyre barotropic quasigeostrophic model for a square basin with $R e=U L / A_{H}$ as the control parameter (from Dijkstra and Katsman, 1997). Drawn (dotted) branches indicate stable (unstable) steady states, whereas the Pitchfork (Hopf) bifurcation points are indicated by squares (triangles).

(small $R e$ ), the anti-symmetric double-gyre flow (at label a in Fig. 3) is a unique state. When lateral friction is decreased, this flow becomes unstable at the pitchfork bifurcation $P_{1}$ (near $R e=30$ ) and two branches of stable asymmetric states appear for smaller values of $A_{H}$ (larger $R e$ ). The solutions on these branches (at locations $b$ and d) have the jet displaced either southward or northward and are exactly symmetrically related for the same value of $R e$ (streamfunction plots of these solutions can be found in Dijkstra and Katsman, 1997).

The important point from Fig. 3 is that multiple stable asymmetric equilibria (here steady flows) can occur under a symmetric wind-stress forcing. The mechanism of this symmetry breaking is due to shear instability and has been explained in detail in Dijkstra and Katsman (1997).

\subsection{Subsequent transitions}

The asymmetric states which arise from $P_{1}$ also become unstable at larger values of $R e$ due to the occurrence of Hopf bifurcations. The first Hopf bifurcation, $H_{1}$ in Fig. 3, is associated with the destabilization due to a so-called Rossbybasin mode. These modes can be described by a sum of free Rossby waves where the coefficients are chosen such that the boundary conditions are satisfied. For the gravest Rossby basin mode, the period is about 20 days. At a second Hopf bifurcation, $\mathrm{H}_{2}$ in Fig. 3, the asymmetric state destabilizes to a mode which has an interannual period and the perturbations strengthen and weaken the eastward jet during both phases of the oscillation. These interannual, so-called gyre modes do not have their origin in the spectrum of the linear operator related to free Rossby-wave propagation. Simonnet and Dijkstra (2002) clarified the spectral origin of the gyre

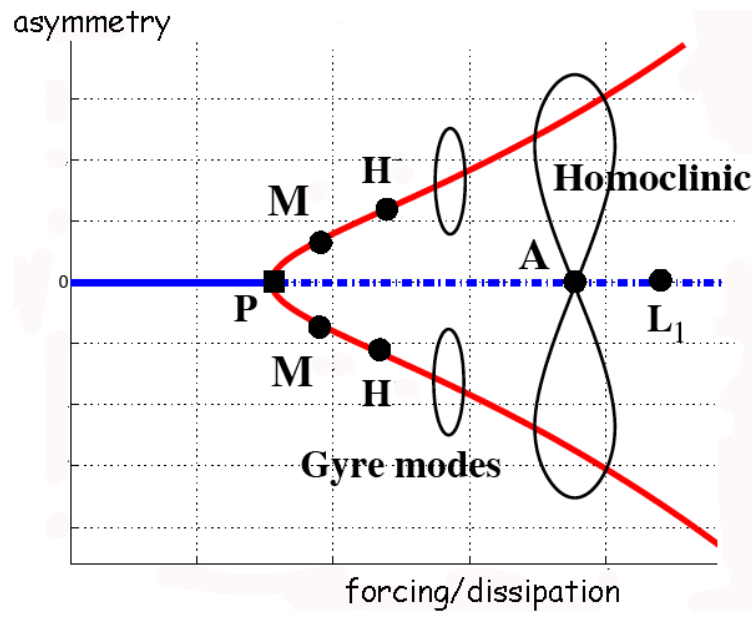

Fig. 4. Schematic bifurcation diagram of the solutions of the barotropic vorticity equation, plotted in terms of a measure of the asymmetry of the solutions versus either wind-stress intensity or the Reynolds number $R e$ (from Simonnet et al., 2005).

mode, presented a physical mechanism of its propagation and showed the relation between the gyre mode and stationary Rossby waves (see also Primeau, 2002).

The connection between the first pitchfork bifurcation $P$, the gyre modes and the occurrence of homoclinic bifurcations was clarified in Simonnet et al. (2005) and an overview of the bifurcation behavior leading to the homoclinic orbit is shown in Fig. 4. The symmetry-breaking pitchfork bifurcation $P$ is responsible for the asymmetric states. The lowfrequency gyre modes arise at so-called merging points $M$ and they obtain a positive growth factor at the Hopf bifurcations $H$. Subsequently, the periodic orbits arising from these Hopf bifurcations on both asymmetric branches connect with the unstable anti-symmetric steady state at the point $A$; this gives rise to a homoclinic orbit. The type of homoclinic orbit depends on the eigenvalues associated with the linear stability of the symmetric state at the connection point $A$ (Wiggins, 1990). In case there are only real eigenvalues, there is a homoclinic connection of Lorenz-type and when the second and third eigenvalue form a complex-conjugate pair, there is a homoclinic bifurcation of Shilnikov type. Simonnet et al. (2005) show that both types can occur and that the Shilnikov type is more likely to occur at small $A_{H}$, in accordance with the results in Meacham (2000) and Nadiga and Luce (2001).

The important point from Fig. 4 is that low-frequency variability arises spontaneously due to Hopf bifurcations and homoclinic bifurcations, even under a steady wind stress and without mesoscale eddies arising through baroclinic instability. In the regime beyond the homoclinic transitions, variability with a decadal time scale is found to be robust in these models (McCalpin and Haidvogel, 1996; Chang et al., 2001; Nauw et al., 2004). 


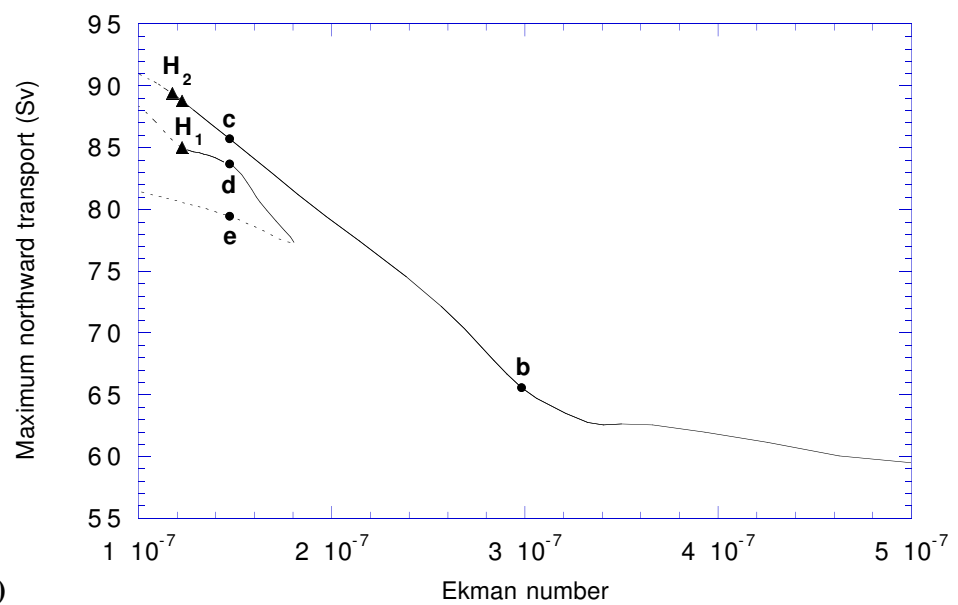

(a)

$$
\text { Ekman number }
$$

(b)

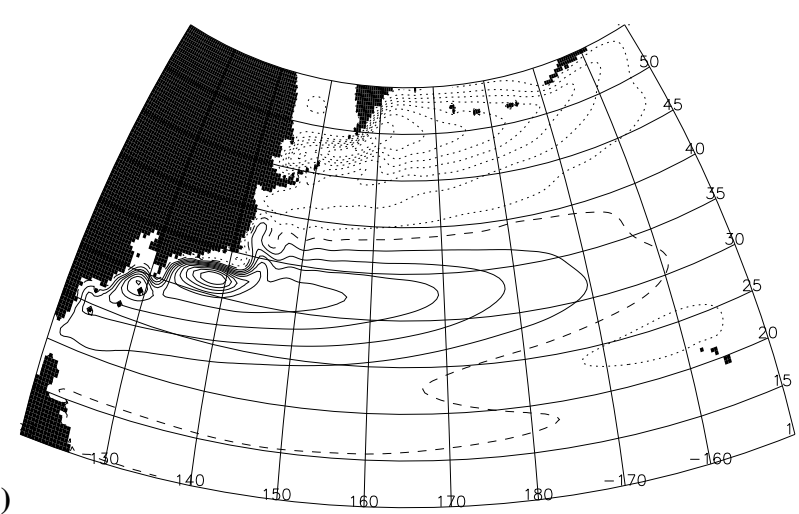

(c)

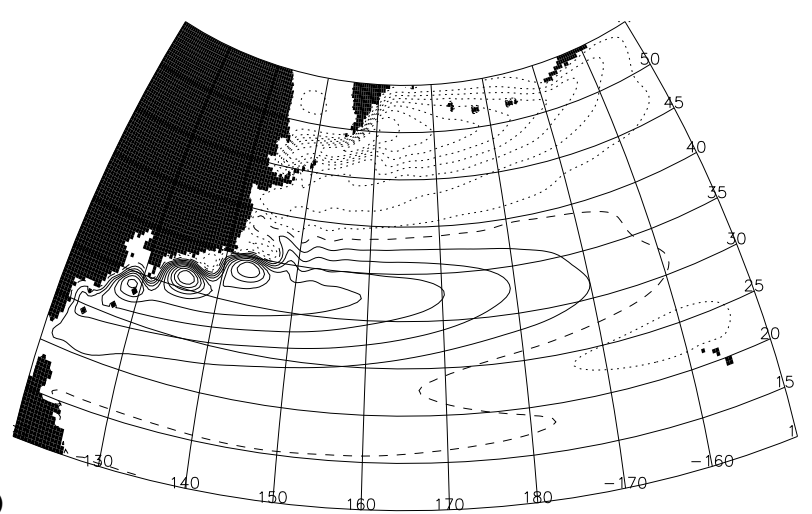

Fig. 5. (a) Bifurcation diagram for the barotropic shallow-water model on the North Pacific domain with the Ekman number $E=A_{H} /\left(2 \Omega r_{0}^{2}\right)$ as control parameter. Drawn (dotted) branches indicate stable (unstable) steady states, whereas the Hopf bifurcation points are indicated by triangles. (b) Contour plot of sea-surface height deviations for the steady state solution at location c on the upper branch in (a). (c) Contour plot of sea-surface height deviations for the steady state solution at location d on the upper branch in (a). The contour levels are scaled with respect to the maximum value of the field (from Schmeits and Dijkstra, 2001).

\subsection{Multiple equilibria in a "realistic" shallow-water model}

The results in the previous section were for quasi-geostrophic models in rectangular basins and one might wonder what happens in reduced-gravity shallow-water model using a more realistic basin geometry and annual-mean observed winds. This issue was investigated in Schmeits and Dijkstra (2001) who performed numerical bifurcation studies for a part of the North Pacific basin $\left[120^{\circ} \mathrm{E}, 150^{\circ} \mathrm{W}\right] \times$ $\left[10^{\circ} \mathrm{N}, 55^{\circ} \mathrm{N}\right]$ using a horizontal resolution of about $1 / 2^{\circ}$ in a barotropic shallow-water model.

As control parameter, the Ekman number $E=A_{H} /\left(2 \Omega r_{0}^{2}\right)$ is used where $r_{0}$ is the radius of the earth and $\Omega$ its angular velocity (for model equations and parameter values, see Schmeits and Dijkstra, 2001). The bifurcation diagram (Fig. 5a), where the maximum northward volume transport (in Sv) is plotted versus $E$, shows a perturbed pitchfork bi- furcation and clearly provides evidence that multiple equilibria exist when the lateral friction is small enough. The perturbed pitchfork arises because the mid-basin symmetry is obviously broken by the geometry and the wind forcing. Note that there is quite a range of Ekman numbers where two equilibria are (barotropically) stable.

The stationary solution at location b (not shown) in Fig. 5a displays a Kuroshio path south of Japan with two recirculation gyres, and an extended jet near Japan similar to the elongated Kuroshio flow. A stable stationary solution on the upper branch (location c) is shown in Fig. 5b for $\left(E=1.5 \times 10^{-7}\right)$, which is in the multiple equilibria regime. It displays a Kuroshio path south of Japan which is different from either the elongated or contracted states. Compared to the solution at location b, to which it is continuously connected, the anti-cyclonic recirculation gyre to the south of Japan has intensified and has caused the Kuroshio to deviate from the coast. 


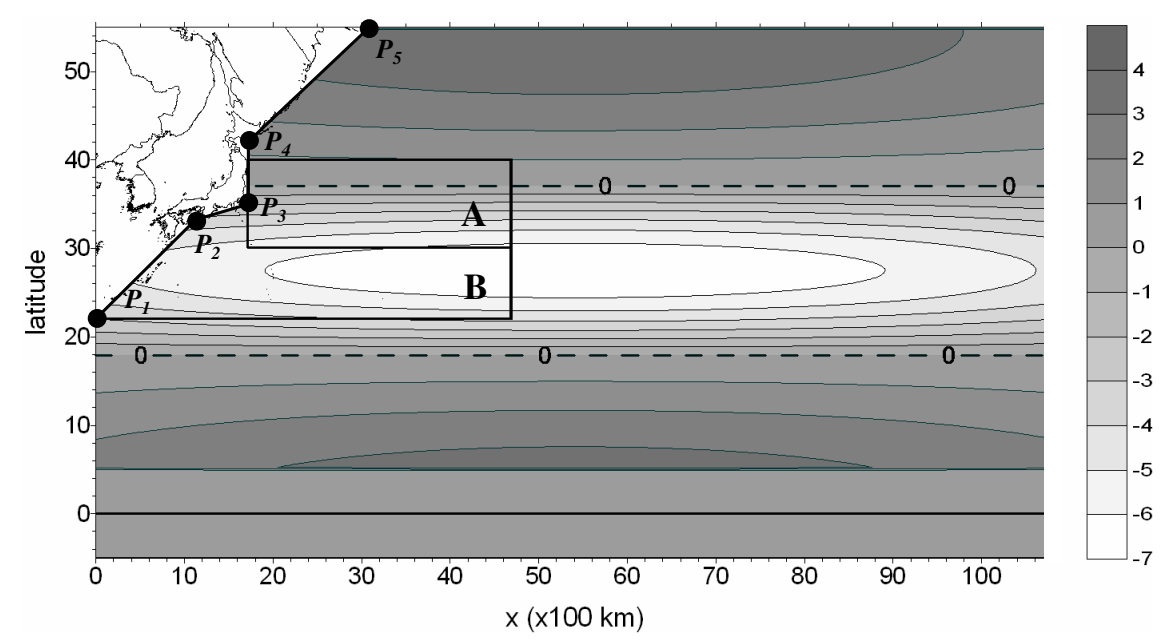

Fig. 6. Domain of integration and contour map of the curl of the wind stress (units in $10^{-8} \mathrm{Nm}^{-3}$ ) used to force the model (from Pierini, 2006).

The second branch of solutions exists only for $E<1.8 \times 10^{-7}$, which is the position of the saddle-node bifurcation on this branch. The solution at $E=1.5 \times 10^{-7}$ (Fig. 5c) displays a Kuroshio path south of Japan, with three recirculation gyres, and a large meander near Japan similar to the contracted state. The third steady state at $E=1.5 \times 10^{-7}$ (location e) is unstable and the Kuroshio has different separation behavior than for the other states.

The important point from Fig. 5 is that multiple equilibria are still present in the more realistic case and that the flow patterns differ in the separation and meandering behavior of the Kuroshio, in particular in the number of recirculation gyres. The circulation patterns outside the region of the western boundary current are very similar for each solution.

\subsection{Transient behavior in a "realistic" double-gyre shallow water model}

In Pierini (2006), the reduced-gravity shallow water equations forced by a steady wind forcing derived from climatological winds are used to study low-frequency KE variability. The governing equations of the model are:

$$
\begin{aligned}
\frac{\partial \boldsymbol{u}}{\partial t}+(\boldsymbol{u} \cdot \nabla) \boldsymbol{u}+f \boldsymbol{k} \wedge \boldsymbol{u} & =-g^{\prime} \nabla \tilde{\eta}+\frac{\boldsymbol{\tau}}{\rho H}+A_{H} \nabla^{2} \boldsymbol{u}-\gamma \boldsymbol{u}|\boldsymbol{u}| \\
\frac{\partial \tilde{\eta}}{\partial t}+\nabla \cdot(H \mathbf{u}) & =0
\end{aligned}
$$

where $\boldsymbol{k}=(0,0,1), \boldsymbol{\tau}=\left(\tau^{x}, 0,0\right)$ and $\boldsymbol{u}=(u, v, 0)$ is the horizontal velocity vertically averaged in the upper active layer, $\tilde{\eta}$ is the interface displacement (positive downwards), $H=D+\tilde{\eta}$ is the upper layer thickness, where the undisturbed layer thickness is $D=500 \mathrm{~m}$, the density of the upper layer is $\rho=1.0235 \mathrm{~g} / \mathrm{cm}^{3}$ and in the reduced gravity the relative variation of density between the two layers is $\Delta \rho / \rho=0.0045$. The reduced-gravity approximation is very appropriate, see the Figs. 4-5 in Qiu et al. (2006), and the value of $g^{\prime}=g \Delta \rho / \rho$ fits well with their observations. Moreover, $\gamma=5 \times 10^{-4} \mathrm{~m}^{-1}$ parameterizes the interfacial friction. Finally, the spatial resolution and time step are $\Delta x=\Delta y=20 \mathrm{~km}$ and $\Delta t=20 \mathrm{~min}$, respectively. The domain of integration is shown in Fig. 6 which also contains a plot of the wind-stress curl. The fundamental importance of the schematic coastline introduced in the western part of the ocean basin and the large zonal width of the latter is discussed by Pierini (2008).

In Pierini et al. (2009), model results for a value of $A_{H}=220 \mathrm{~m}^{2} \mathrm{~s}^{-1}$ are compared to observations (see that article for a full account of this comparison). In Fig. 7, the annual-averaged SSH fields of Qiu and Chen (2005) (color images) are compared with the SSH fields obtained from model results (grayscale images) according to a synchronization of model/observations where year 1993 is identified with model year 157. In 1993 the KE is in the elongated state and corresponding model solution $(t=157 \mathrm{yr})$ agrees well for the first large anticyclonic meander in position, shape and strength. After 11 years a similar situation is reached (year 2004, $t=168 \mathrm{yr}$ ), and the agreement is now even better for the first and second anticyclonic meanders and for the cyclonic meander south of Japan. For intermediate times, the variability found in the model results is in agreement with the observations. The disruption of the elongated state, accompanied by the disappearance of the two main anti-cyclonic meanders, occurs in less than 1 year (from year $1994, t=158 \mathrm{yr}$ to year $1995, t=159 \mathrm{yr}$ ).

Also the observed KE path length $L_{\mathrm{KE}}$ and mean latitudinal position $\bar{\phi}$ of the upstream KE axis (both defined in Qiu and Chen, 2005) were compared with the corresponding model results for $A_{H}=220 \mathrm{~m}^{2} \mathrm{~s}^{-1}$ in Pierini et al. (2009), as shown in Fig. 8. The behavior based on data can be summarized as follows (Fig. 8a and b): (i) during the 

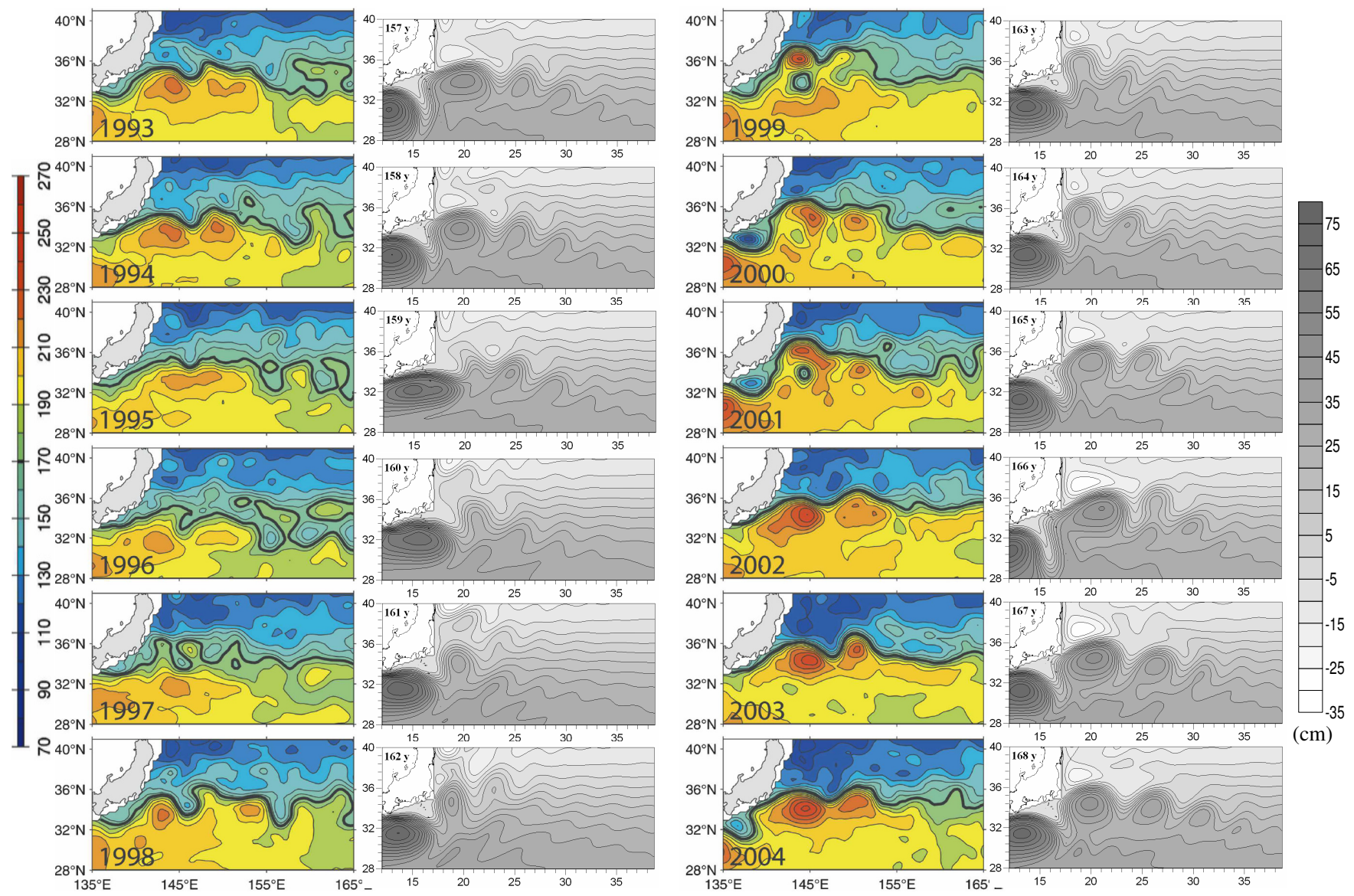

Fig. 7. Maps of yearly averaged SSH field computed from altimeter data (adapted from Fig. 2 of Qiu and Chen, 2005). Panels in gray scale: snapshots of the SSH fields computed from model data at the beginning of each year. A correspondence of year 1993 with model year 157 is made (from Pierini et al., 2009).

elongated state (roughly covering the periods $1993-1995$ and 2002-2005) $L_{\mathrm{KE}}$ and $\bar{\phi}$ are both weakly varying about their mean value; (ii) during the recharging (transition) phase of the relaxation oscillation, that lasts about 7 years and connects those two periods, $L_{\mathrm{KE}}$ yields a much larger (high frequency) variability while $\bar{\phi}$ shows a clear positive trend until approximately the end of the transition. The model data for two successive cycles (Fig. 8c-f) shows the same qualitative behavior and also an acceptable quantitative agreement both as far as the timing and the amplitudes are concerned.

The bifurcation behavior of the model solutions with $A_{H}$ as control parameter was also investigated in Pierini et al. (2009). Figure 9 shows a partial bifurcation diagram obtained by performing a large number of forward time integrations. For each value of $A_{H}$ the curves with labels min and max give the range within which $E_{A}$ (the kinetic energy per unit mass integrated over the sector A of Fig. 6) varies after spinup. For $A_{H}<230 \mathrm{~m}^{2} \mathrm{~s}^{-1}$ the two pairs of curves $b_{\min }-b_{\max }$ and $c_{\min }-c_{\max }$ are associated with the spontaneous switching of the trajectory between two equilibrium flows. It was found that in the interval (between the two dashed lines) $A_{H}=240 \mathrm{~m}^{2} \mathrm{~s}^{-1}$ down to $A_{H}=235 \mathrm{~m}^{2} \mathrm{~s}^{-1}$, the system undergoes an impressive change in behavior. There is a transition from weak amplitude irregular oscillations at $A_{H}=240 \mathrm{~m}^{2} \mathrm{~s}^{-1}$ (corresponding to gyre-mode variability) to a much larger amplitude relaxation-type oscillation at $A_{H}=235 \mathrm{~m}^{2} \mathrm{~s}^{-1}$.

In Fig. 10a, the abrupt transition from a small amplitude oscillation to a large amplitude relaxation oscillation is evidenced in the time series. In the $E_{A}-E_{B}$ phase plane (Fig. 10b), where $E_{B}$ is the kinetic energy of the flow over a region B south of Japan, the view is even more dramatic; for $A_{H}=240 \mathrm{~m}^{2} \mathrm{~s}^{-1}$ (blue curve) the trajectory occupies a relatively small area in state space while for $A_{H}=235 \mathrm{~m}^{2} \mathrm{~s}^{-1}$ the trajectory suddenly explores a new high energy area in state space. This change in behavior can be illustrated in more detail by considering the Probability Density Function (PDF) in the $E_{A}-E_{B}$ phase plane. Figure 10c shows that the PDF is confined in a restricted region around the unstable fixed point for $A_{H}=240 \mathrm{~m}^{2} \mathrm{~s}^{-1}$, and then expands for $A_{H}=235 \mathrm{~m}^{2} \mathrm{~s}^{-1}$, embracing regions well beyond the original basin of attraction, characteristic of a homoclinic bifurcation. 

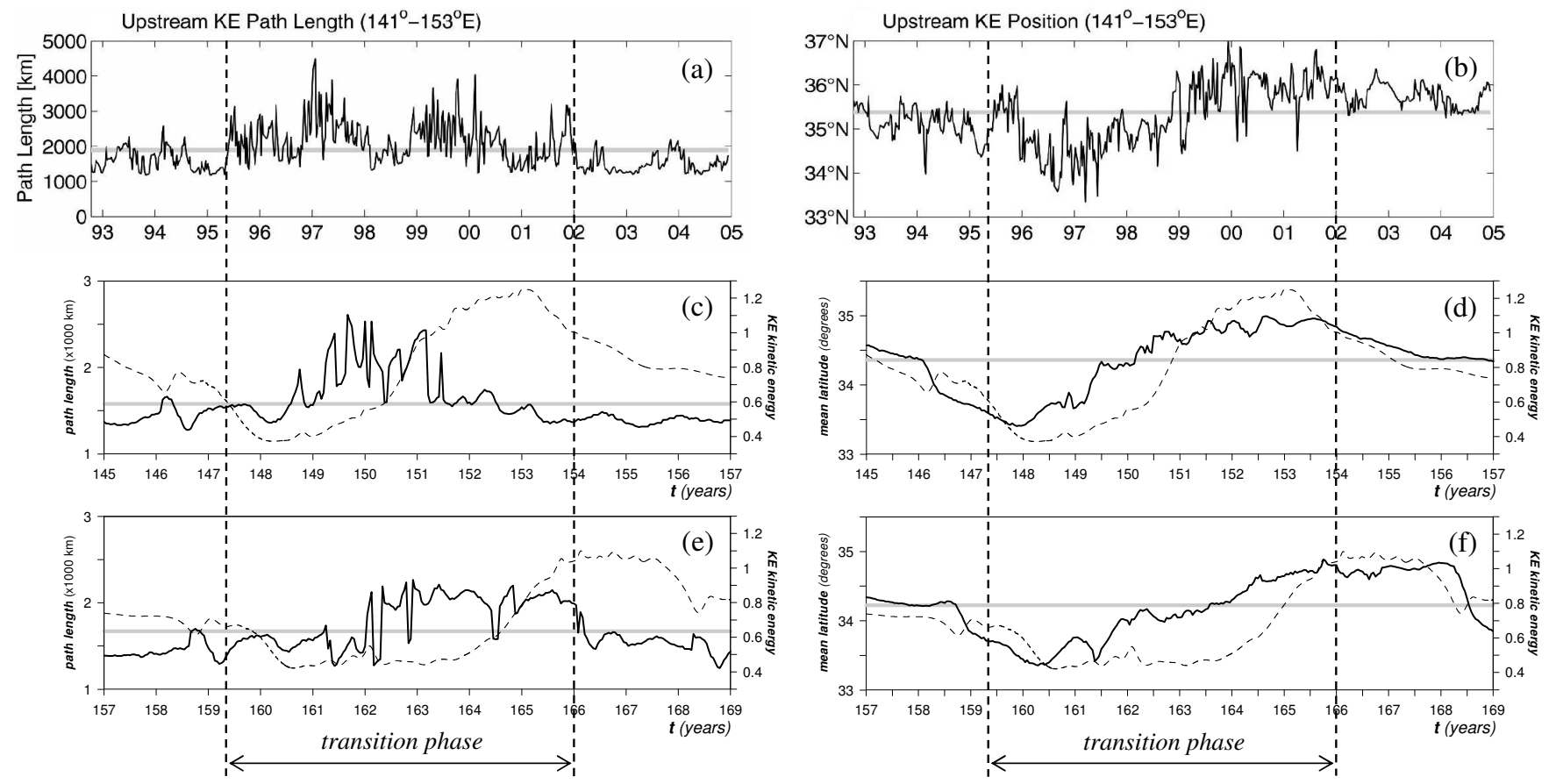

Fig. 8. (a) Upstream KE path length integrated from $141^{\circ} \mathrm{E}$ to $153^{\circ} \mathrm{E}$ computed from altimeter data (adapted from Fig. 4 of Qiu and Chen, 2005); (c, e) same quantity computed from model data for two successive cycles, respectively. (b) Latitudinal position of the KE averaged from $141^{\circ} \mathrm{E}$ to $153^{\circ} \mathrm{E}$ (adapted from Fig. 4 of Qiu and Chen, 2005); (d, f) same quantity (drawn curves) computed from model data for two successive cycles, respectively; the thick gray line is the mean value of each quantity. In each panel c-f, the thick gray line is the mean value, and the dashed line is the kinetic energy $E_{A}$ per unit mass integrated over a sector A which covers the KE region (units in $10^{13} \mathrm{~m}^{5} \mathrm{~s}^{-2}$ ). The time interval between the two vertical dashed lines corresponds to the transition phase (from Pierini et al., 2009).

The important point of this subsection is that lowfrequency KE variability, resembling observations both qualitatively and quantitatively, is found in a reduced-gravity shallow-water model under steady forcing. This variability very likely results from a homoclinic transition similar to those found in the quasi-geostrophic models of the doublegyre circulation (Sect. 3.3).

\section{Discussion and conclusions}

From the analysis presented in this note it is clear that two forms of low-frequency variability (V1 and V2 heretofore) exist in the KE system. There is (V1) a highly nonlinear, spatially sharp, frontal bimodal behavior characterized by two completely different contracted and elongated jet states alternating chaotically on a decadal time scale through very distinctive and asymmetric transitions between them. Moreover, there is (V2) a weaker, essentially linear, spatially broad field of westward-traveling Rossby waves. These two forms of variability appear to be synchronized, at least during the 15 year-period 1993-2007, as documented by Qiu and Chen (2009, in press) through altimeter data.
In the Rossby wave view (Sect. 2) the bimodal weakening (strengthening) in the KE jet and recirculation gyre is considered to be consistent with westward propagation of negative (positive) Rossby sea surface height anomalies (hindcast using linear vorticity dynamics) generated in the eastern North Pacific by varying winds (Qiu and Chen, 2005); so, in this view, V1 is to some extent identified with V2. An appropriate scaling of the fully nonlinear quasigeostrophic vorticity equation shows, however, that both the Kuroshio and the KE, in its various stages of the observed bimodality, are highly nonlinear dynamical features (as usual for western boundary currents and for their extensions), so, the method followed in the Rossby wave view (Sect. 2) of analyzing the KE bimodal variability by decomposing it into a climatological mean jet (determined from observations) plus a Rossby wave signal solution of a linear model does not appear to be adequate in principle, because the modulating bimodal signal can hardly be described by the linearized dynamics and would interact nonlinearly with the mean flow. So, it is not surprising to find that the results of such a modeling approach are unable to account for the observed bimodality (Fig. 2). The same results do show, however, an interesting synchronization of the Rossby wave signal V2 with the observed bimodal variability V1, posing an interesting fundamental issue (see below). 


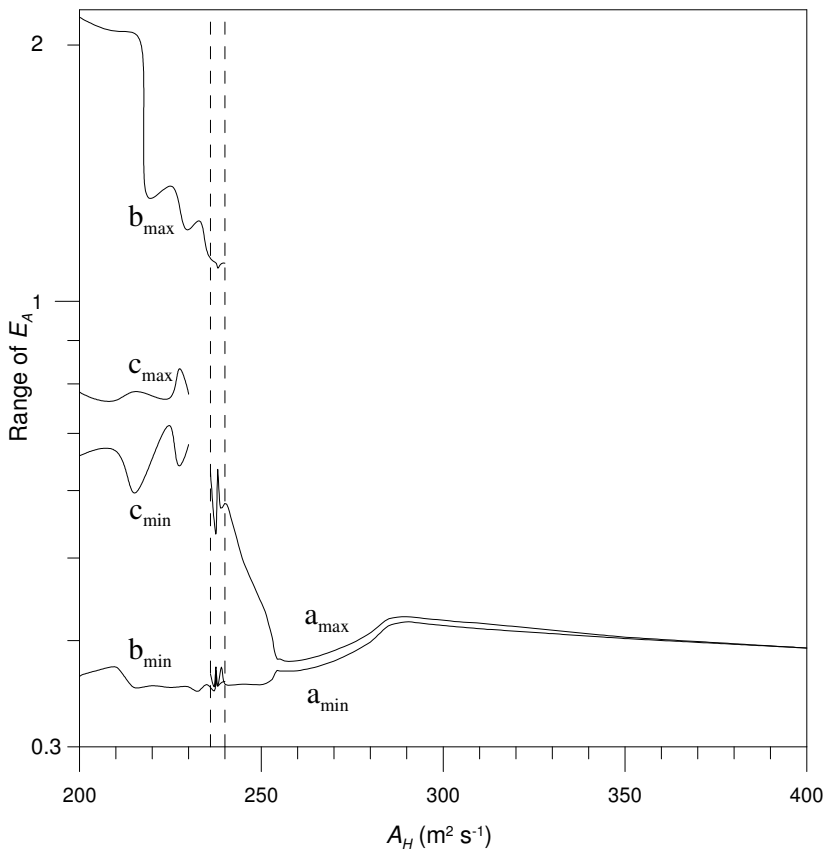

Fig. 9. Bifurcation diagram, with the control parameter given by the lateral eddy viscosity $A_{H}$. The curves with labels min and max give the range within which $E_{A}$ varies after spinup. For $A_{H}<230 \mathrm{~m}^{2} \mathrm{~s}^{-1}$ the two pairs of curves $b_{\min }-b_{\max }$ and $c_{\min }-c_{\max }$ are associated with the spontaneous switching of the trajectory between two equilibrium flows (from Pierini et al., 2009).

In the intrinsically generated variability view (Sect. 3), a fully nonlinear shallow water model (Sect. 3.5) has proved successful in reproducing quantitatively (within the limits of an idealized model implementation) the most salient features of the KE bimodality V1. Another interesting aspect that we here want to emphasize is that the time-mean state obtained from the modeled low-frequency variability (which is substantially different from both the contracted and the elongated states) is in good agreement with the observed climatology of Teague et al. (1990), as shown in Sect. 3 of Pierini (2006). So, in the intrinsically generated variability view, an appropriate dynamical model provides also a sufficiently realistic mean jet as a byproduct of the time-dependent response. This is conceptually a great advantage over the linearized analysis typical of the Rossby wave view.

Support for the intrinsic generation of V1 is also provided by ocean general circulation model studies. Through an empirical orthogonal function analysis of an eddy-resolving model (OFES) hindcast, Taguchi et al. (2007) decomposed the variability into a meridionally broad mode and a frontalscale mode: the first is recognized to be associated with Rossby wave activity while, for the second, nonlinear intrinsic oceanic mechanisms are suggested to be likely. In fact, a climatological run was shown to produce an amount of frontal variability in the KE region that is comparable to that
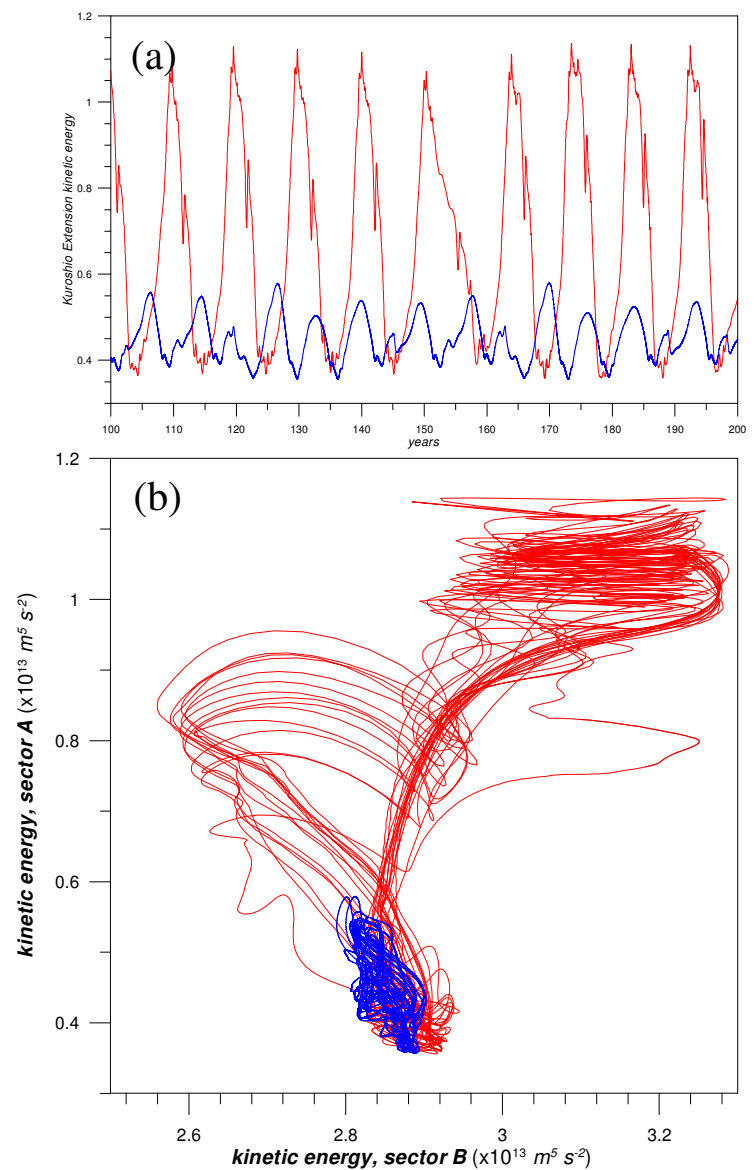

(c)

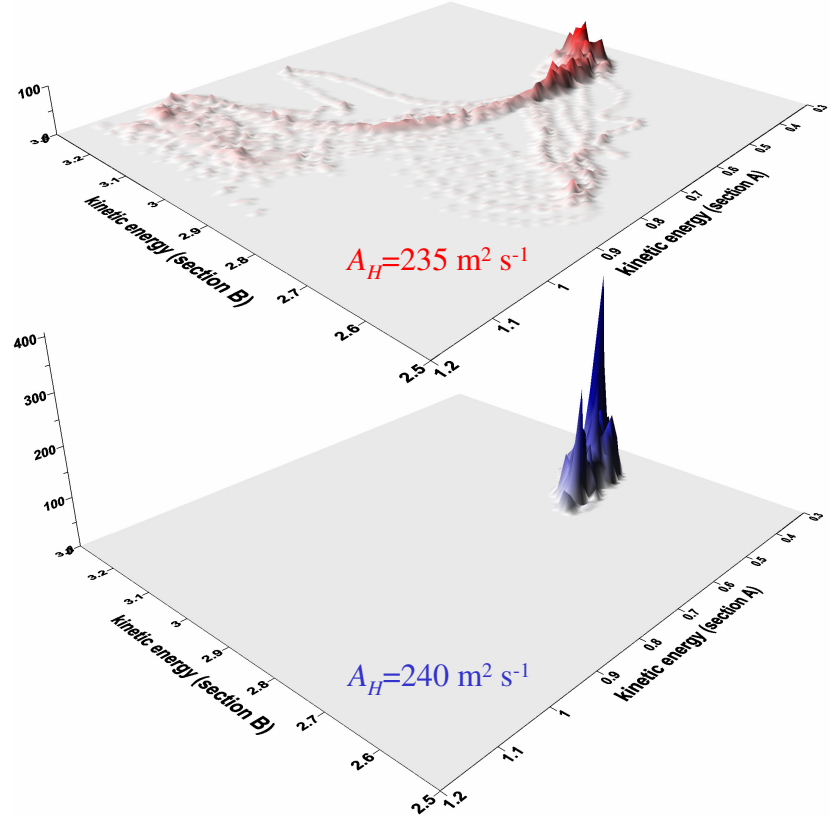

Fig. 10. (a) Time series of the kinetic energy $E_{A}$ and (b) projection of flow trajectory onto the $E_{B}-E_{A}$ plane, where $E_{B}$ is the kinetic energy of the flow over a region $\mathrm{B}$ south of Japan, for $A_{H}=240 \mathrm{~m}^{2} \mathrm{~s}^{-1}$ (blue line) and $A_{H}=235 \mathrm{~m}^{2} \mathrm{~s}^{-1}$ (red line). (c) Probability density function $P$ on the $E_{B}-E_{A}$ plane for $A_{H}=240 \mathrm{~m}^{2} \mathrm{~s}^{-1}$ (lower panel) and $A_{H}=235 \mathrm{~m}^{2} \mathrm{~s}^{-1}$ (upper panel; energy units in $10^{13} \mathrm{~m}^{5} \mathrm{~s}^{-2}$ ). Figure is from Pierini et al. (2009). 
obtained with a hindcast run. In conclusion, the analyses of Sect. 3 strongly support the hypothesis that the KE bimodal variability V1 is generated by intrinsic oceanic mechanisms.

However, even assuming that this is true, an intriguing question remains open: why is the Rossby wave signal V2 basically in synchrony with the bimodal variability V1? At least two explanations may be conjectured after assuming that V1 is intrinsically generated. In the first, the initiation of the chaotic bimodal cycle and its subsequent evolution are little affected by the atmospheric variability; on the other hand, the very strong sea surface temperature anomalies associated with the bimodal cycle (Qiu, 2000; Qiu and Miao, 2000) would induce in the atmosphere a large-scale response that, in turn, would generate the Rossby wave signal. Under these circumstances, the latter is expected to be correlated with V1. In this rather extreme view, the PDO and NPGO (North Pacific Gyre oscillations), that are considered to be the cause of V2 (Qiu and Chen, 2009, in press), would be strongly affected, if not controlled, by V1.

In a second explanation, in which the internal mechanisms play a less decisive role, the wind variability and the corresponding Rossby wave signal trigger the KE bimodal cycle (which implies the observed correlation between the two), but the subsequent evolution is again determined by the internal ocean dynamics. This scenario is similar to that envisaged by Taguchi et al. (2007) who suggested that the Rossby wave signal could control the KE bimodal variability through a spatial reorganization of the KE flow (from a meridionally broad to a frontal scale structure) thanks to internal variability of the ocean system. As a matter of fact, Pierini (2009) showed that the modeled KE bimodality discussed in Sect. 3.5 is associated with an internal mode that, in a certain dissipative range, needs a (red) wind noise to be excited.

In conclusion, with the observational KESS program, the use of altimetry data and the detailed analysis of model behavior, we may not be far from a theory of the low-frequency KE variability which satisfactorily explains the existence of the contracted and elongated state and the decadal time scale transitions between them. We finally suggest that the use of quantities such as the KE path length $L_{\mathrm{KE}}$ and the mean latitudinal position $\bar{\phi}$ of the upstream KE axis proposed by Qiu and Chen (2005) can serve as very useful indicators for identifying the KE bimodality in the analysis of model results (as it was the case in Pierini, 2006 and Pierini et al., 2009).

Acknowledgements. We are glad to acknowledge valuable discussions with Bo Qiu, and thank him for providing the Figs. 1 and 2 of this paper. We also thank the Scientific Committee of the workshop "Nonlinear Processes in Oceanic and Atmospheric Flows" for organizing such an enjoyable workshop in Bilbao (Spain) during July 2008.

Edited by: A. M. Mancho

Reviewed by: M. Spydell and two other anonymous referees

\section{References}

Cessi, P. and Ierley, G. R.: Symmetry-breaking multiple equilibria in quasi-geostrophic, wind-driven flows, J. Phys. Oceanogr., 25, 1196-1205, 1995.

Chang, K.-I., Ghil, M., Ide, K., and Lai, C.-C. A.: Transition to aperiodic variability in a wind-driven double-gyre circulation model, J. Phys. Oceanogr., 31, 1260-1286, 2001.

Dijkstra, H. A.: Nonlinear Physical Oceanography: A Dynamical Systems Approach to the Large Scale Ocean Circulation and El Niño., Kluwer Academic Publishers, Dordrecht, The Netherlands, 2000.

Dijkstra, H. A. and De Ruijter, W. P. M.: Finite Amplitude Stability of the Wind-Driven Ocean Circulation, Geophys. Astrophys. Fluid, 83, 1-31, 1996.

Dijkstra, H. A. and Katsman, C. A.: Temporal variability of the Wind-Driven Quasi-geostrophic Double Gyre Ocean Circulation: Basic Bifurcation Diagrams, Geophys. Astrophys. Fluid, 85, 195-232, 1997.

Donohue, K., Watts, D. R., Tracey, K., Wimbush, M., and Park, J.: Program Studies the Kuroshio Extension, EOS T. Am. Geophys. Un., 89, 161-162, 2008.

Jiang, S., Jin, F.-F., and Ghil, M.: Multiple equilibria and aperiodic solutions in a wind-driven double-gyre, shallow-water model, J. Phys. Oceanogr., 25, 764-786, 1995.

Mantua, N. J., Hare, S., Zhang, Y., Wallace, J. M., and Francis, R. C.: A Pacific interdecadal climate oscillation with impacts on salmon production, B. Am. Meteorol. Soc., 78, 1069-1079, 1997.

McCalpin, J. D. and Haidvogel, D. B.: Phenomenology of the low-frequency variabiliity in a reduced gravity quasi-geostrophic double-gyre model, J. Phys. Oceanogr., 26, 739-752, 1996.

Meacham, S. P.: Low frequency variability of the wind-driven circulation, J. Phys. Oceanogr., 30, 269-293, 2000.

Munk, W.: On the wind-driven ocean circulation, J. Meteorol., 7, 79-93, 1950.

Nadiga, B. T. and Luce, B.: Global bifurcation of Shilñikov type in a double-gyre model, J. Phys. Oceanogr., 31, 2669-2690, 2001.

Nauw, J., Dijkstra, H. A., and Simonnet, E.: Regimes of lowfrequency variability in a three-layer quasi-geostrophic model, J. Mar. Res., in press, 2004.

Pedlosky, J.: Geophysical Fluid Dynamics, 2nd edn., SpringerVerlag, New York, 1987.

Pierini, S.: A Kuroshio Extension system model study: decadal chaotic self-sustained oscillations, J. Phys. Oceanogr., 36, 16051620, 2006.

Pierini, S.: On the crucial role of basin geometry in double-gyre models of the Kuroshio Extension, J. Phys. Oceanogr., 38, 13271333, 2008.

Pierini, S.: Coherence resonance in a double-gyre model of the Kuroshio Extension, J. Phys. Oceanogr., in press, 2009.

Pierini, S., Dijkstra, H. A., and Riccio, A.: A nonlinear theory of the Kuroshio Extension Bimodality, J. Phys. Oceanogr., 39, 22122229, 2009.

Primeau, F. W.: Multiple equilibria and low-frequency variability of the wind-driven ocean circulation, J. Phys. Oceanogr., 32, 22362256, 2002.

Qiu, B.: Interannual variability of the Kuroshio Extension System and its impact on the wintertime SST field, J. Phys. Oceanogr., 30, 1486-1502, 2000. 
Qiu, B. and Chen, S.: Variability of the Kuroshio Extension Jet, Recirculation Gyre and Mesocale Eddies on decadal time scales, J. Phys. Oceanogr., 35, 2090-2103, 2005.

Qiu, B. and Chen, S.: Eddy-Mean flow interaction in the decadallly modulating Kuroshio Extension system, Deep-Sea Res., in press, 2009.

Qiu, B. and Miao, W.: Kuroshio Extension variability and forcing of the Pacific Decadal Oscillations: responses and potential feedback, J. Phys. Oceanogr., 33, 2465-2482, 2000.

Qiu, B., Hacker, P., Chen, S., Donohue, K. A., Randolph Watts, D., Mitsudera, H., Hogg, N. G., and Jayne, S. R.: Observations of the subtropical mode water evolution from the Kuroshio Extension System Study, J. Phys. Oceanogr., 36, 457-473, 2006.

Schmeits, M. J. and Dijkstra, H. A.: Bimodality of the Kuroshio and the Gulf Stream, J. Phys. Oceanogr., 31, 2971-2985, 2001.

Simonnet, E. and Dijkstra, H. A.: Spontaneous generation of lowfrequency modes of variability in the wind-driven ocean circulation, J. Phys. Oceanogr., 32, 1747-1762, 2002.

Simonnet, E., Dijkstra, H. A., and Ghil, M.: Homoclinic bifurcations in the quasi-geostrophic double-gyre circulation, J. Mar. Res., 63, 931-956, 2005.

Speich, S., Dijkstra, H., and Ghil, M.: Successive bifurcations in a shallow-water model applied to the wind-driven ocean circulation, Nonlin. Processes Geophys., 2, 241-268, 1995, http://www.nonlin-processes-geophys.net/2/241/1995/.
Stommel, H.: The westward intensification of wind-driven ocean currents, EOS T. Am. Geophys. Un., 29, 202-206, 1948.

Sverdrup, H. U.: Wind-driven currents in a baroclinic ocean with application to the equatorial current in the eastern Pacific, P. Natl. Acad. Sci. USA, Wash., 33, 318-326, 1947.

Taft, B. A.: Characteristics of the flow of the Kuroshio south of Japan, in: Kuroshio, physical aspects of the Japan current, edited by: Stommel, H. and Yoshida, K., Univ. Washington Press, Seattle, USA, 1972.

Taguchi, B., Xie, S.-P., Mitsudera, H., and Kubokawa, A.: Response of the Kuroshio Extension to Rossby waves associated with the 1970 s climate shift in a high resolution ocean model, J. Climate, 18, 2979-2995, 2005.

Taguchi, B., Xie, S.-P., Schneider, N., Nonaka, M., Sasaki, H., and Sasai, Y.: Decadal variability of the Kuroshio Extension: Observations and an eddy-resolving model hincast, J. Climate, 20, 2357-2377, 2007.

Teague, W. J., Carron, M. J., and Hogan, P. J.: A comparison between the generalized digital environment model and Levitus climatologies, J. Geophys. Res., 95, 7167-7183, 1990.

Veronis, G.: An analysis of the wind-driven ocean circulation with a limited number of Fourier components, J. Atmos. Sci., 20, 577 593, 1963.

Wiggins, S.: Introduction to Applied Nonlinear Dynamical Systems and Chaos, Springer-Verlag, Heidelberg-Berlin, Germany, 1990. 